\title{
Fortgeschrittenes Nierenzellkarzinom Was tun, wenn der erste Tyrosinkinasehemmer versagt?
}

\begin{abstract}
Der mTOR-Inhibitor Everolimus ist in der Lage, das progressionsfreie Überleben von Patienten mit fortgeschrittenem Nierenzellkarzinom nach Versagen der First-Line-Therapie mit einem Tyrosinkinaseinhibitor im Vergleich zu Placebo signifikant zu verlängern. Dies zeigt die Zulassungsstudie RECORD-1 (REnal Cell cancer treatment with Oral RAD001 given Daily).
\end{abstract}

Die Europäische Kommission hat im August den mTOR-Inhibitor Everolimus (RAD001, Afinitor ${ }^{\circledR}$ ) zur Therapie von Patienten mit fortgeschrittenem Nierenzellkarzinom (RCC) zugelassen, bei denen es während oder nach einer gegen VEGF (Vascular Endothelial Growth Factor) gerichteten Therapie zu einer Krankheitsprogression gekommen ist. Gegen VEGF sind z.B. Tyrosinkinaseinhibitoren gerichtet, die in Deutschland als Standardtherapie gelten. Wie Kurt Miller, Berlin, erklärte, kann Everolimus im Vergleich zu Placebo in Kombination mit der bestmöglichen supportiven Versorgung der Patienten das mediane progressionsfreie Überleben auf mehr als das Doppelte verlängern (4,9 vs. 1,9 Monate, $\mathrm{p}<0,001$, Abb. 1). Dabei wird das Risiko eines Krankheitsprogresses bzw. das Sterberisiko um $67 \%$ verringert. Damit wird eine wesentliche Therapielücke in der Therapie von RCCPatienten geschlossen.
Primärer Studienendpunkt war das progressionsfreie Überleben. Eingeschlossen wurden 416 Patienten, die im Verhältnis 2:1 auf Verum bzw. Placebo randomisiert wurden. Die in die Placebogruppe randomisierten Patienten hatten nach dem Fortschreiten der Erkrankung die Möglichkeit, eine unverblindete Behandlung mit Everolimus zu erhalten. Daher war die Studie nicht geeignet, einen Unterschied im Gesamtüberleben der Patienten zu dokumentieren.

\section{Überzeugender Benefit}

Umso bemerkenswerter ist es jedoch, so Miller, dass sich dennoch ein so deutlicher Unterschied im progressionsfreien Überleben belegen ließ, der dann auch zu einem durch das wissenschaftliche Komitee veranlassten, vorzeitigen Studienabbruch führte. Der oral verfügbare mTOR-Inhibitior war im Allgemeinen gut verträglich.

Die europäischen Leitliniengremien (EAU und EORTC-
GU) haben bereits auf die positiven Ergebnisse der RECORD1-Studie reagiert und Everolimus mit höchster Evidenzstufe als medikamentöse Standardtherapie beim fortgeschrittenen RCC nach der Behandlung mit einem Tyrosinkinaseinhibitor empfohlen. Eine ähnliche Empfehlung hat auch das US-amerikanische NCCN ausgesprochen.

TUK

Quelle: Novartis Oncology Launch-Presseveranstaltung am 17. August 2009 in Nürnberg (Veranstalter: Novartis)

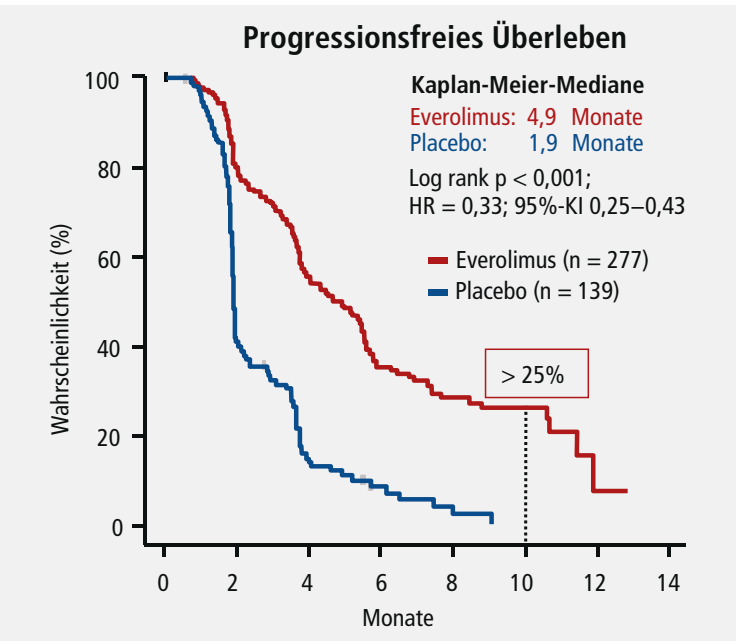

Abbildung 1: RECORD-1-Studie: Everolimus verlängerte beim fortgeschrittenen Nierenzellkarzinom das progressionsfreie Überleben nach der ersten Tyrosinkinaseinhibitor-Therapie signifikant (Nach Motzer RJ et al. Lancet 2008;372:449-56 (Update ASCO GU 2009).
Rituximab jetzt auch bei vorbehandelter CLL zugelassen

Seit Kurzem steht Rituximab (MabThera ${ }^{\circledR}$ ) in Europa auch zur Anwendung bei Patienten mit rezidivierter oder refraktärer chronisch lymphatischer Leukämie (CLL) zur Verfügung. Die Zulassung basiert auf den Daten der REACH-Studie, der mit 552 Patienten größten internationalen randomisierten Studie bei vorbehandelter CLL. Patienten, die mit Rituximab in Kombination mit Fludarabin und Cyclophosphamid behandelt wurden, lebten im Median zehn Monate länger ohne Fortschreiten der Krebserkrankung als bei alleiniger Chemotherapie (30,6 vs. 20,6 Monate). Zur CLL-Erstbehandlung ist Rituximab in Kombination mit Chemotherapie bereits der Standard.
Generisches Irinotecan

Mit Irinomedac ${ }^{\circledR}$ steht ein generisches Irinotecanpräparat in den Wirkstärken 40,100 und $300 \mathrm{mg}$ zur Verfügung. Der Topoisomerase-IHemmer ist für die Therapie des fortgeschrittenen Kolorektalkarzinoms zugelassen. Die Wirkstärke $300 \mathrm{mg}$ passt optimal zu gängigen Therapieschemata wie FOLFIRI. 